\title{
ESPACIOS DE RELACIÓN Y PARTICIPACIÓN COMUNITARIA EN UN PUERTO YUCATECO: PESCA, PRÁCTICAS RELIGIOSAS Y "POLILLA"
}

\author{
María Teresa Castillo Burguete y \\ María Dolores Viga de Alva *
}

\section{Introducción: \\ La participación comunitaria, en la mira de las agencias para el desarrollo}

$\mathrm{E}$

n décadas recientes, las agencias mundiales dedicadas al desarrollo han puesto es pecial énfasis para obtener la participación de las comunidades, con lo cual han pretendido poner en marcha proyectos, a fin de transformar sus condiciones de pobreza y atender los problemas sociales (Uphoff, 1995). Han expresado este interés en acciones realizadas en países de África, Asia y América Latina, a través de programas promovidos tanto por organizaciones gubernamentales como por no gubernamentales, operados a niveles local, estatal, nacional e internacional. A lo largo de las últimas décadas los proyectos de desarrollo han sido usados, con frecuencia, como vía para financiar el crecimiento y el cambio inducidos (Cernea, 1995:33), canalizando a través de ellos gran cantidad de recursos como parte de los intentos por cambiar las condiciones materiales de diversos grupos sociales.

María Teresa Castillo Burguete y María Dolores Viga de Alva, Centro de Investigación y de Estudios Avanzados del IPN, Unidad Mérida.
En el ámbito mundial, el énfasis puesto en el tema de la participación comunitaria no es casual; es producto del seguimiento de proyectos de desarrollo que no han alcanzado metas y objetivos satisfactorios. Una de las causas identificadas es que la colaboración de individuos y comunidades locales ha sido insuficiente. Si bien es cierto que la participación no puede considerarse una panacea, su importancia es tal que las vías para obtenerla en el nivel comunitario han sido una preocupación presente en diversos ámbitos. Por ello, la participación (Rifkin et al, 1988), la participación popular (Rahnema, 1997; Fals Borda, 1988), la participación comunitaria, el poder de la comunidad (Hawley and Wirt, 1974), también han sido temas de análisis y discusión en diversos foros y trabajos, como parte de los factores que intervienen en las políticas de desarrollo y de atención a la pobreza.

Como parte de los programas de desarrollo, las actividades de autoayuda para introducir e inducir cambios en las prácticas populares han requerido de la participación comunitaria. Una propuesta sobre algunas formas para obtenerla fue promovida por el Departamento de Estado norteamericano. A través del Community Services Staff, Go- 
biernos y organismos internacionales (SAHOP s.f.; ops/OMs, 1978, 1984; Banco Mundial, 1997: 1152; Banco Mundial 2001) han identificando que los programas de combate a la pobreza debían contener el ingrediente ineludible de la participación de los grupos objetivo. Los antecedentes expuestos nos permiten concordar en que:

...se ha reconocido cada vez más la importancia de la participación, no sólo para obtener más influencia en la toma de decisiones por parte de los beneficiarios del programa, sino también para asegurar que la infraestructura y los servicios programáticos alcancen su objetivo original. La verdadera participación de los grupos aldeanos en la programación de las inversiones y la toma de decisiones continúa siendo limitada (Uphoff, 1995:540).

En su momento, también la Organización de las Naciones Unidas (ONU), en la Conferencia sobre Asentamientos Humanos efectuada en Vancouver en 1976, reconoció la participación comunitaria como uno de los mecanismos centrales para lograr el desarrollo social de los países del llamado tercer mundo y de las regiones pobres y marginadas (Pearse y Stiefel, 1980). Al respecto, Pliego (1996) señala que desde entonces, el concepto de participación comunitaria ha venido acuñando un prestigio tal que su inclusión en los programas sociales parece legitimarlos por el solo hecho de indicar una base participativa, a tal grado que un programa social específico puede tener problemas técnicos de diverso tipo, incluso puede no estar bien diseñado y conceptuado, pero si es evaluado como una propuesta fuertemente participativa, entonces tendrá superados gran parte de los obstáculos para su apoyo financiero.
En las postrimerías del siglo xx los resultados desalentadores del paradigma más reciente del desarrollo condujeron a que un grupo de expertos se reuniera en Washington para buscar respuestas acerca de "...ंpor qué han aumentado los niveles de pobreza en América Latina durante los últimos 10 años?, ¿por qué ha crecido el abismo entre ricos y pobres?” (Funaro, 1997: 48).

Gobiernos e instituciones para el desarrollo coinciden al considerar la participación como un ejercicio libre, deseable, para lograr los cambios en las poblaciones que sufren pobreza, pero también ha llegado a convertirse en un lema política y económicamente atractivo, un instrumento de eficacia, fuente de inversión y aprovisionamiento de fondos para proyectos. $\mathrm{Al}$ incorporar procesos participativos en los proyectos de desarrollo, se pretende evitar las fallas del pasado. Un ejemplo de ello se da en el campo de la salud, donde se han hecho esfuerzos por tratar de tener un conocimiento cercano de la 'realidad del campo', que trabajadores de la salud extranjeros y la burocracia gubernamental no tienen; ubicar redes de relaciones, esenciales para la marcha del proyecto y la inversión a largo plazo en el área rural; obtener, de igual forma, la cooperación en la escena local, de organizaciones capaces de llevar a cabo actividades de desarrollo (Rahnema, 1997).

La participación local se ha considerado el eslabón más débil de la cadena y el interés por lograr esa participación no es exclusivo de organizaciones con planes dirigidos al llamado tercer mundo: también se encuentra en la mira de organizaciones europeas que operan para las poblaciones de ese continente, en un intento para saber cómo afrontar el cambio económico y social a escala del barrio (Chanan, 1993). ${ }^{3}$ 
La participación comunitaria suele ser requerida como una necesidad externa a la comunidad, promovida como una vía a través de la cual se espera que miembros de grupos locales o comunidades se involucren en un proyecto o programa dirigido a disminuir la pobreza, a promover el desarrollo o a transformar las condiciones de salud de aquellos pueblos o comunidades de que se trate. Esta es una de las formas de participación, pero existen otras evidenciadas en trabajos realizados en el área maya (Redfield y Villa Rojas, 1934; Villa Rojas, 1946; 1987; Brown, 1993, Castillo, 2001), que describen prácticas relacionadas con la vida comunitaria, con su organización social, política y religiosa, y permiten identificar aspectos presentes de la participación de los individuos en grupos, expresadas en diversos ámbitos, como parte de la vida diaria, arraigadas en el caudal de las costumbres y de la vida cotidiana de la población. Estas últimas son minimizadas o ignoradas y suelen contener elementos de gran importancia para comprender las formas de participación locales.

Aun cuando ya han transcurrido varias décadas, sigue vigente la recomendación de conocer las comunidades con las cuales se pretende trabajar, de realizar estudios de base (Community Services Staff, 1953; Burgman y Ooijens, 1989) con los cuales se conozca la situación económica, sociocultural en que se encuentran los futuros participantes, así como los posibles impedimentos para su participación, especialmente sus deseos y necesidades en relación con el proyecto. También se plantea la necesidad de reconocer e incentivar la participación de todos los interesados, efectuar cambios en las actitudes y encontrar los estímulos adecuados, de tal forma que tanto las organizaciones no gubernamentales (ONG's) como el sector público encuentren puntos de colaboración. Se debe considerar al sector primario como socio esencial, ya que resulta fundamental tener organizaciones basadas en la comunidad (Smith, 1997).

Pese a la importancia de los planteamientos expuestos, a los esfuerzos realizados y al tiempo transcurrido, aún se desconoce mucho del tejido de la vida comunitaria. Las propuestas de cambio todavía son hechas desde cierta exterioridad y es frecuente que se hagan conociendo poco de esas formas internas de organización, de su construcción cotidiana, de la estructura social de las poblaciones, de sus principios, valores, ideales y formas específicas de ver el mundo, de su cultura. En este sentido la diversidad cultural existente en los pueblos hace necesario primero conocerlos y, solamente después, sugerir o planear las acciones más adecuadas con la comunidad. Para poder llevar a cabo esa tarea, las ciencias sociales, especialmente la antropología, tienen mucho que aportar. El trabajo pionero de Gamio (1922) expresaba la importancia de las ciencias sociales en los estudios previos para el desarrollo de regiones $(\mathrm{Nahmad}$, 1995).

\section{Espacios, entorno construido y procesos sociales}

Si bien la comunidad y la participación no se dan necesariamente en espacios fijos, algunas de las actividades de los participantes tienen lugar en espacios físicos, llevando a la construcción de espacios sociales más abstractos. Estos han sido estudiados como clave para el entendimiento de los procesos sociales, donde las conductas humanas se manifiestan como relaciones entre cultura y ambiente construido (Rapoport $\left.{ }^{4}, 1990\right)$. Este planteamiento nos motivó para estudiar los escenarios o lugares de reunión comunitarios, la forma en 
que son creados, las personas involucradas en ellos y las temáticas tratadas en las reuniones.

La importancia que tiene conocer las relaciones y las transformaciones que establecen hombres y mujeres con su entorno y la manera en que lo influyen también ha sido preocupación de estudiosos formados en diversas disciplinas. Resaltan las contribuciones en los estudios geográficos sobre México, sus diversos exponentes y escuelas, entre las que se encuentran las mexicanas y sus influencias provenientes de la geografía humana y la geografía histórica francesas (Hoffmann y Salmerón, 1997). También las perspectivas de la historia y antropología han sido incorporadas junto con la geografía (Aboites, 1995). En la perspectiva de la antropología mexicana:

...el sustento espacial ha sido una preocupación permanente. Tanto las regiones de refugio, como los sistemas hidráulicos, la producción campesina, los asentamientos urbanos, obreros e industriales y los sistemas regionales de dominación política, tienen un soporte espacial característico y una elaboración cultural propia (Hoffmann y Salmerón, 1997:16).

Si bien Rapoport (1994) plantea que fueron muy pocos los investigadores que consideraron en sus estudios el papel de la cultura y la importancia de ver comparativamente el comportamiento humano en su relación con el entorno, en la actualidad se ha constituido en una subárea de los estudios sobre entorno y comportamiento (Hollahan, 2000), y no se puede eludir la importancia del papel de la cultura para comprender las relaciones entre la gente y los espacios que ocupan.

Comprender el concepto de entorno construido ayuda a explicar el uso del espacio, según Lawrence y Low (1990) un concepto abstracto empleado para describir los productos de la construcción humana. En un sentido amplio se refiere a toda alteración física del ambiente natural debida a ese tipo de construcción. Incluye, de manera general, las formas construidas creadas por los seres humanos para resguardar, definir y proteger sus actividades. Estas formas también incluyen espacios definidos y delimitados, pero no necesariamente considerados, tales como las áreas no cubiertas de un conjunto de edificios, una plaza o una calle; pueden contener monumentos o sitios, como santuarios, que no necesariamente comprenden la actividad.

Los entornos construidos (Rapoport, 1994; Lawrence y Low, 1990) constituyen mucho más que construcciones que abarcan gran parte de los llamados entornos "naturales", tales como campos, huertos, jardines, caminos, debido al gran peso que la cultura tiene para comprender las relaciones entre la gente y sus entornos. Geógrafos y antropólogos conciben el espacio como un ámbito de negociación cotidiana entre los actores, redefinido y conceptuado de diversas formas, vinculado con las relaciones sociales, los flujos económicos y las características físicas del territorio y también con las representaciones culturales de cada pueblo (Hoffmann y Salmerón, 1997).

En el espacio social se desarrolla un sistema social definido, en el sentido propuesto por Stacey (1969), como el conjunto de instituciones interrelacionadas que cubren los aspectos de la vida social, familiar, religiosa, jurídica, etcétera, así como los sistemas de creencias asociados. Cada uno de los aspectos mencionados pueden considerarse como sistemas en sí mismos y a la vez como partes de un sistema social. En este sentido, en el espacio social las prácticas pueden ser diversas, no solamente inclusivas, sino también de segregación 
(Schnell, 1999). La institución social es definida por Stacey (1969), siguiendo a Ginsberg, como los usos reconocidos y establecidos que gobiernan las relaciones entre individuos o grupos.

Además de ser el contenedor material de los procesos sociales, el espacio social es también un elemento que influye en la estructuración misma de la sociedad. En este sentido, Hall (1972) sostiene que el hombre [y la mujer] ha creado una nueva dimensión cultural y la relación que establece esa dimensión es tal que, tanto él como su medio participan en un modelado mutuo. De esta manera, la distribución, el cómo se usan los ámbitos, los lugares o el espacio, los significados que tienen para las culturas específicas que los usan, han sido parte de los aspectos estudiados a través de la antropología del espacio.

De acuerdo con Hall, los seres humanos han creado prolongaciones materiales de la territorialidad, indicadores visibles e invisibles, que llevan a identificar tres tipos de espacios: de caracteres fijos, semifijos y el espacio informal. El espacio de caracteres fijos es uno de los moldes donde se forja gran parte del comportamiento, es “... uno de los modos fundamentales de organizar las actividades de los individuos y los grupos. Comprende manifestaciones materiales tanto como normas ocultas, interiorizadas, que rigen el comportamiento cuando el hombre se mueve sobre la tierra" (Hall, 1972:127-28). Un ejemplo de ello es que si bien los edificios son una expresión de pautas de caracteres fijos, se encuentran agrupados de formas características y las divisiones en su interior están dadas de acuerdo con normas determinadas culturalmente.

Los espacios de caracteres semifijos (Hall, 1972), pueden cambiar de acuerdo con ciertas pautas, teniendo en cuenta que lo que en una civilización es espacio de caracteres fijos, puede serlo de semifijos en otra y viceversa. La categoría de experiencia espacial es, quizá, la más importante para el individuo porque entran en ella las distancias sostenidas en los encuentros con otras personas y son, en gran parte, la conciencia del espacio exterior ajeno. Este espacio es llamado "informal" debido a que no es declarado. Estas normas tienen límites distintos y un significado tan hondo que forman parte esencial de la cultura.

Si bien Rapoport (1990) confiere importancia a los conceptos de cultura y entorno construido, también advierte sobre la dificultad que implica comprender y explicar la relación entre ambos en un nivel tan general y abstracto; por ello sugiere desagregarlos, a fin de estudiar las relaciones entre sus componentes más específicos. El entorno puede desagregarse en formas de organización de espacio, tiempo, significado y comunicación, en lo que llama sistema de actividades. Para el caso concreto de las relaciones entre pesca y actividades religiosas en la participación comunitaria es necesario explorar esas cuatro formas de organización, como una vía para comprender la influencia que puede tener el entorno en ella.

Como la noción de actividades no siempre es evidente, debe esclarecerse en términos de la relación de prácticas de la cultura y también en términos de cuatro aspectos de las actividades, que van desde 1) aspectos instrumentales, los cuales manifiestan en su mayoría la naturaleza de las actividades a través de, 2) cómo son asociadas al sistema, 3) cómo son llevadas a cabo las actividades, 4) su significado, sus aspectos más latentes (Rapoport, 1990; 1990a). Una consecuencia del punto dos de ese análisis da lugar al tercer punto principal, en el sentido de que no se puede analizar las actividades por sí solas, sino como partes 
integrantes de un sistema de actividades. Más aún, los otros aspectos de las actividades también juegan un papel importante en los escenarios usados. Una forma de analizarlas es mediante los mecanismos mayores o superiores, los cuales vinculan los escenarios con la gente y los significados de sus actividades.

Un escenario tampoco puede ser considerado de manera separada, sino como parte de un sistema, de tal forma que el sistema de actividades actualmente ocurre en un sistema de escenarios; organizados en una variedad de formas complejas, no solamente en el espacio sino también en el tiempo y en otras formas, todas relacionadas con la cultura. En este sentido, sucede que una parte del sistema influye fuertemente en lo que pasa o no en otra parte de sí mismo (Rapoport, 1990), como veremos en actividades relacionadas con la pesca y las actividades religiosas del puerto de estudio.

\section{Participación comunitaria y espacios de relación entre pesca, religión y política}

"No participan, son apáticos, sólo les interesa qué cosas se les va a dar y a veces ni eso", fueron algunas de las opiniones que escuchamos de diversas personas, que han efectuado trabajo comunitario e, incluso, de varios de los miembros de dos comunidades, en la costa de Yucatán, México.

Los comentarios previos pueden llevarnos a pensar que los miembros de esa comunidad son apáticos, las propuestas externas no son de su interés, no reconocen tener "problemas" o éstos no son de la incumbencia de quienes no son miembros de la comunidad, las convocatorias no son las adecuadas, que en la vida comunitaria ya todo está organizado como algo dado y es inne- cesario tomar decisiones, etcétera; sin embargo, como hemos visto, la participación y en particular la comunitaria, es parte de la misma cultura y tradición. Además, en todas las comunidades podemos observar ámbitos a los cuales concurren cuando es necesario, y la organización emerge. En este sentido es importante identificar cuáles son los elementos de unión que regulan sus relaciones y les permiten actuar a través de los mecanismos de organización y de toma de decisiones existentes en la comunidad, los cuales no necesariamente aparecen a simple vista.

En trabajo de campo relacionado con otro proyecto de investigación (Ortega y Dickinson, 19915; Dickinson y Ortega, 1994), realizamos visitas a la comunidad de El Puerto, las cuales llevaron a plantearnos las preguntas que motivaron este estudio y a planearlo de tipo cualitativo. Enmarcarlo lleva a recordar parte del camino abierto por Boas (1911) y Malinowski (1973) en la antropología social, al plantear el trabajo de campo como un esfuerzo antropológico (Taylor, S. y Bodgan, R. 1996), necesario y enriquecedor.

Enfocamos la investigación cualitativa en diversos métodos, involucrando un acercamiento interpretativo a los sujetos y problemas. Para ello requerimos reunir y usar una variedad de materiales empíricos que describen rutinas y momentos problemáticos y significativos en la vida de los individuos como estudios de caso, experiencia personal, introspectiva, historias de vida, entrevistas, observaciones y textos visuales. Buscamos respuestas para explicar cómo se crea la experiencia social y la forma en que tiene un significado específico, desplegando una amplia gama de métodos interconectados, con el fin de obtener una mejor base sobre el tema y su manejo (Denzin y Lincoln, 1994). 
El lugar de estudio fue El Puerto ${ }^{6}$, y se encuentra localizado en la barra arenosa costera de Yucatán. Cuenta con una diversidad de ecosistemas que le permiten actividades económicas diversificadas (Mapa 1), tales como la pes- ca, la producción de sal, el cultivo de plantas de coco para ornato y de coco de agua, la acuicultura, el turismo y, en menor escala, la horticultura y la milpa (Castillo y Dickinson, 1994).

\section{Mapa 1: Recursos naturales existentes en El Puerto, Yucatán}

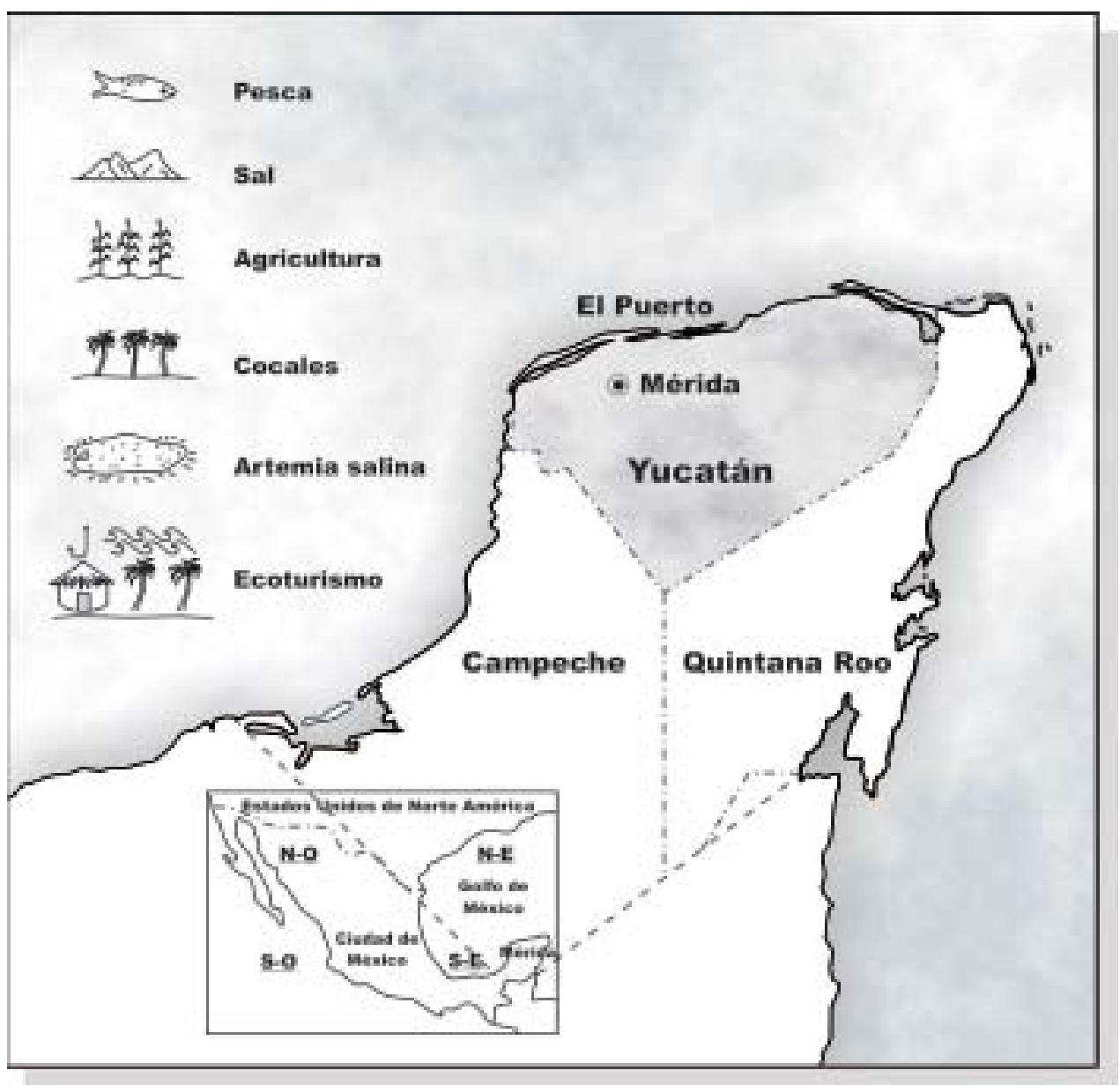

Elegimos este lugar porque es una comunidad con la cual podríamos tener relación directa con casi todos sus integrantes, mantener contacto en la observación de reuniones, en sus ámbi- tos y escenarios, la vida cotidiana y entrevistar con facilidad a interlocutores clave. Contábamos con datos básicos obtenidos en años anteriores, que formaron parte de un estudio más amplio. 
También teníamos buenas relaciones con varios miembros de la comunidad.

En el lugar viven aproximadamente 586 habitantes, 277 mujeres y 309 hombres, agrupados en 141 familias. Varias de las características generales de este puerto y sus habitantes son representativas de otros lugares de la costa yucateca donde encontramos que la pesca es una actividad importante para el sustento familiar, al igual que el renglón de servicios, para cubrir parte de las necesidades de los visitantes que llegan durante las épocas de semana santa y el verano.

Al realizar la investigación solemos separar actividades productivas de aquellas disímiles como las de índole religiosa. Esta práctica refiere a un orden elemental y seguramente así seguiremos haciéndola, sin embargo, y aunque podría parecer extraño en las reuniones mensuales de los socios de la Unidad de Producción Pesquera Ejidal El Puerto (UPPEEP) analizaron cuestiones religiosas, y en la iglesia católica, un lugar de celebración religiosa, trataron temáticas relacionadas con la pesca. Ciertamente existe una separación entre las actividades productivas y religiosas, pero los resultados de esta investigación marcan la existencia de puntos de intersección entre ambos que resultan de gran interés para comprender la participación comunitaria. El entramado de estas relaciones no es tan visible y constituye uno de los puntos clave de esa vida. De ello trataremos en este apartado.

La población de El Puerto es mayoritariamente católica (70.5\%), este hecho se expresa en su participación cotidiana, seis de cada diez de los eventos observados en el ciclo comunitario anual ${ }^{7}$ estuvieron relacionados con esta iglesia, lo que me dio como resultado que seis de cada diez participantes, lo hicieron en eventos relacionados con ella. $\mathrm{Al}$ analizar las frecuencias de participación por género y tipo de participantes en actividades relacionadas con esta religión, encontramos que las mujeres realizaban tres cuartas partes de ella y los hombres una. En El Puerto son las mujeres quienes organizan y llevan a cabo gran parte de la vida comunitaria, no solamente a nivel religioso, pues en el total de actividades comunitarias ellas participaron el doble que sus compañeros varones. Los adultos varones que participan en los rituales católicos son principalmente de la tercera edad u hombres jóvenes que acuden con su esposa.

Las actividades religiosas católicas trascienden el mero recinto de la iglesia, la sacristía y el atrio. También se transforman en espacios rituales para la celebración religiosa católica los hogares de los feligreses, las calles, el parque, la comisaría municipal, la cancha de baloncesto, el centro de salud, otros lugares relacionados con actividades productivas tales como el puerto de abrigo, el mar y la sede de la UPPEEP, la agrupación de pescadores. La religiosidad católica se expresa en casi todos los lugares de la comunidad.

De todos los lugares usados para el análisis de la totalidad de eventos ${ }^{8}$ de participación comunitaria, los efectuados en la iglesia católica ocuparon la primera posición, seguida de la cancha de baloncesto; en tercera y cuarta posición, las casas particulares y la comisaría municipal, respectivamente. Tanto la iglesia católica como la cancha de baloncesto y la comisaría municipal son lugares de acceso a todo el que así lo desee, excepto cuando existen marcadores semifijos que indican lo contrario, al formar parte de las regulaciones culturales que los miembros de la comunidad interpretan correctamente. Un ejemplo de ello lo constatamos en el uso de la cancha de baloncesto que pasa a ser un lugar de celebración de ritos de paso, en eventos tales como bodas y fiestas de quince años, don- 
de, si bien aparentemente sigue abierto al que pase por sus alrededores, resulta accesible solamente para aquellos que hubieran sido invitados verbalmente o por escrito. Estos acuerdos son respetados por los lugareños.

Las casas de los miembros de El Puerto también se abren para dar lugar a celebraciones rituales generalmente signadas por la fe católica. Este espacio se abría al ámbito de la religión al celebrar actividades como el círculo bíblico, el cenáculo o los rezos, algunos asociados a otro rito de paso: la muerte. Actividades como las mencionadas congregaban a vecinas, familiares y amigas. La presencia de mujeres y niños era notable, así como la casi nula presencia de varones adultos. En El Puerto son las mujeres quienes ponen en marcha el mecanismo para organizar gran parte de las actividades relacionadas con la iglesia católica, en la iglesia limpian el local, arreglan el altar con flores y veladoras, mantienen cortinas y manteles limpios, chapean? el atrio, obtienen recursos para su funcionamiento al organizar verbenas donde venden antojitos, dulces que ellas preparan y refrescos embotellados. $\mathrm{Si}$ el sacerdote quiere realizar alguna actividad relacionada con el calendario religioso o distribuir materiales para su estudio, ellas son los contactos. A través de los centros familiares organizados por iniciativa del sacerdote, también están a cargo del calendario ritual a través de los centros familiares, cuyos nombres también están vinculados con la iglesia: una mujer es responsable de cada uno de ellos.

En los eventos de la iglesia católica existe un intenso patrón discursivo relacionado con la vida comunitaria, más allá de ser grupo religioso, son llamados a hermanarse, a cooperar entre sí, a luchar por la paz y el bienestar de todos los que viven en el lugar y en otras partes del mundo. El papel relevante que las mujeres realizan en la vida comunitaria queda nítidamente reconocido por el sacerdote, quien en la víspera de la conmemoración del día de la mujer, hizo el siguiente llamado:

...tenemos que lograr nuestras metas uniendo el esfuerzo de cada uno... "no seamos pesimistas", dice el señor. A veces da tristeza cuando alguien en la comunidad dice "vamos a cambiar" y otros dicen "no vamos a cambiar nada", somos pesimistas. A veces fácilmente le damos la espalda a las cosas, hay gente que por no cambiar apaga las iniciativas de otros, nosotros tenemos que apoyar el proyecto de Dios, que es un pueblo que viva en la justicia y la caridad. Hay hermanos que tienen una iniciativa y los demás comienzan a hacerle la vida imposible, no hay esperanza de fraternidad. Mañana es el día de la mujer, se celebra el día de la mujer, confiar en Dios significa confiar en el prójimo. Si hay algo que las mujeres estén luchando por hacer, no destruyamos lo que están haciendo. Dios es el único que puede transformar, para eso nosotros tenemos que colaborar. Mañana se conmemora el día de la mujer. Tenemos que luchar. También la mujer tiene que tomar participación y entonces el señor verá realizado su proyecto de vida, porque eso quiere Dios para su reino de justicia y de amor.

Al igual que parte de la jerarquía católica reconoce el papel protagónico de las mujeres en la vida comunitaria, ellas, en sus oraciones también atienden el llamado a ser comunidad. Piden por todos los niños, por los jóvenes, por los pescadores, por los señores, por las mujeres, por los enfermos, por los líderes mundiales, por la madre Teresa o por Lady Di, personaje de tierras lejanas, entonces recién fallecida. Este patrón seguido por las mujeres concuerda con su práctica, al dedicar gran parte de su esfuerzo comunitario al beneficio de los ni- 
ños y de su comunidad en general, sin distinguir adscripción política o religiosa.

El otro ámbito que nos ocupa, y con el cual estamos relacionando las actividades religiosas, es el caso de la agrupación de pescadores (UPPEEP). Algunas de sus características indican que son 26 socios, tienen un local propio ubicado al frente de la playa. En la calle principal que marca de sur a norte el asentamiento, ahí se encuentran sus oficinas, bodega y patio. Los socios usan también el puerto de abrigo donde estacionan sus barcos para salir a pescar. Tienen ahí una oficina y realizan la entrega de producto a los compradores de la compañía intermediaria ${ }^{10}$, quienes tienen un local en ese puerto.

Los socios celebran reuniones el primer lunes de cada mes, a las ocho de la noche, en el local que se encuentra en la comunidad. Mientras se reúnen conversan sobre la pesca, su escasez o abundancia, los costos de la carnada, gasolina, cordel y los precios que les estaban pagando por el producto. La afiliación a este grupo está regulada por la vía de sus estatutos, de tal forma que a sus reuniones, formalmente, sólo pueden asistir los socios. Sin embargo la secretaria también suele estar presente y jugar un papel ambiguo, pues mientras los socios reconocen el manejo que tiene de los estados financieros de cada barco y de la unidad en su conjunto, también es blanco de bromas relacionadas con su carácter, por ser muy directa en sus juicios. Los socios piden que sea ella quien realice los informes y no los directivos, "tú sí eres chingona", —le dicen — ella los presenta y aclara cuanta duda manifiestan.

La UPPEEP es una organización de afiliación masculina, sin embargo, en la práctica la secretaria mantenía un lugar prominente. Aunque formalmente sin derecho a voz ni voto, ella opinaba, era escuchada y tenía un peso importante en las opi- niones de los socios. Este caso no parece ser úni$\mathrm{co}$, si tenemos en cuenta que durante entrevistas y conversaciones casuales obtuvimos evidencia de que dos mujeres ahora casadas, y que antes fueron secretarias de la agrupación, tienen no solamente un excelente dominio de ese trabajo administrativo, sino también de la perspectiva de la actividad pesquera y son llamadas para actividades ocasionales de la organización.

Las reuniones comenzaban con la lista de asistencia, pues quienes acumulaban determinado número de faltas quedaban fuera de la asociación, de acuerdo con los estatutos. En seguida daban lectura de los informes financieros de las embarcaciones, de las cantidades que adeudaban a la banca, asuntos relacionados con el estado financiero de la agrupación, con el manejo de equipo, precios de venta, apoyos de los empresarios a quienes les vendían el pescado y el pulpo durante la época de captura.

Durante el desarrollo de las reuniones, los socios participaban activamente, comentando, sugiriendo, proponiendo, preguntando, manifestando acuerdos o desacuerdos sobre los temas tratados. Casi todo el desarrollo de las reuniones era con intervenciones en tono de broma. Hasta los asuntos que parecían más delicados — como las deudas bancarias de la agrupación- eran condimentados con dosis de humor.

Durante las reuniones de la agrupación, también se abría un ámbito de información sobre los eventos ocurridos en la comunidad y los que estaban en proceso. También se fortalecían relaciones de amistad o parentesco, pues varios de los socios tenían lazos de este tipo. Su grado de integración les permitió enfrentar situaciones que parecían de responsabilidad exclusiva del presidente del grupo, ante lo cual los socios expresaban opiniones tales como: “...te estás ahogando en un vaso de 
agua, tú no eres dueño de la sociedad, no estás solo, si hay un problema se reúne el comité, que ayude. Si el comité no es pantalla, está para que funcione." De igual forma establecieron de común acuerdo los precios a los que venderían su producto, teniendo en cuenta también ciertas lealtades al indicar que no podían abandonar al que les había estado ayudando desde tiempo atrás. Señalaban que se debía ver las ventajas y las desventajas de este hecho.

Se identifican y preocupan como colectividad por la situación financiera de su asociación. Buscan y analizan alternativas para obtener apoyos a través de los cuales cambiar ese panorama. La visita de un prominente comerciante de especies marinas a una de sus sesiones propició la oportunidad para externarle sus preocupaciones sobre el apoyo que les había ofrecido. Éste les indicó su interés por apoyar, sin imposiciones porque "esos tiempos ya pasaron, lo que ustedes decidan" - les dijo.

Si bien las temáticas tratadas en la UPPEEP estaban en su gran mayoría relacionadas con la pesca, un mes antes de la fecha de elecciones conversaron sobre aspectos relacionados con "la polilla", refiriéndose a que "estamos en tiempo de polilla", lo que ocasionaba bromas y risas entre los participantes.

La fiesta de la virgen de Fátima el 13 de mayo, patrona del lugar representa uno de los puntos más altos del esfuerzo colectivo comunitario. Después de llevar varios meses observando la participación comunitaria en El Puerto resultó notable la presencia de hombres adultos desde "Las mañanitas", evento que marca el inicio de la fiesta, su presencia en la misa y participación en el paseo por el mar, de donde gran parte de ellos obtienen el sustento. Este parece ser uno de los días más importantes de participación comunita- ria donde se entrelazan los ámbitos religioso y productivo, donde el espacio ritual trasciende la tierra para extenderse hacia el mar, día durante el cual mujeres y hombres adultos, adolescentes y niños se hacen presentes, después de haber contribuido de diversas maneras para preparar la celebración.

En la organización de la fiesta de la virgen advertimos “...fenómenos de gran importancia que no pueden recogerse mediante interrogatorios ni con el análisis de documentos, sino que tienen que ser observados en su plena realidad. Llamémosles los imponderables de la vida real." (Malinowski, 1973). En este sentido, gran parte de la comunidad sabe que los gremios de los días de las vírgenes de Fátima y Guadalupe les corresponde a los miembros de la UPPEEP, que ellos harán la fiesta en su local. El día de la virgen de Fátima llegó, efectuaron el gremio y la fiesta, pero pocos sabían cómo hicieron los pescadores para obtener todo lo necesario para dar comida, bebida y música a los participantes. Más aún, se desconocía cómo se coordinan los diversos participantes para cumplir con una de las costumbres de la comunidad: festejar a su patrona.

En las reuniones sobre la cooperativa, los pescadores también trataban acerca de cómo sacar adelante ese compromiso, dado que tenían severos problemas financieros. En el caso de la fiesta para la virgen de Fátima, la noche previa al inicio de los gremios, los socios analizaron muy preocupados, pero sin perder el sentido del humor, cómo salir adelante con el compromiso, porque significaba realizar gastos y no estaban en condiciones de hacerlo. Primero pensaron dar solamente un jugo y, a decir de algunos socios, la comunidad entendería que sería porque no tenían recursos. Pusieron en juego las redes de relaciones con las que 
cuentan organizaciones como ésta, al recordar que estaban en "tiempo de polilla", y que existían las condiciones para pedir apoyo a los candidatos que contendían por la alcaldía y por una diputación.

En la lista de gremios para el festejo de la virgen de Fátima figura el "de los señores". A ellos les correspondía arreglar el altar, pero la esposa de uno de ellos comentó que ella lo hizo porque él “está viendo lo de mañana." Este señor era también presidente de la UPPEEP, organización a la cual le tocó el gremio junto con la comunidad. Los primeros se encargaron de dar la fiesta y la comida. En su mensaje para los miembros del gremio, el sacerdote inició diciendo que "...la oración la hacemos para pedir por todos los señores de esta comunidad, por su trabajo, aunque no veo ninguno, deben estar trabajando". Su mensaje destacó que anteriormente las comunidades eran pequeñas, diez o doce creyentes, pero ahora hay comunidades de fieles que tienen cuarenta mil, por ejemplo en Mérida, pero:

...la Iglesia tiene una red que ayuda a mantener la unidad de la fe, la comunidad de la Iglesia. La Iglesia ha vivido durante siglos, se ha organizado, tiene una estructura, eso la ha ayudado a permanecer, a diferencia de otras agrupaciones. María ayuda a su hijo en su ministerio, por la intercesión de ella pedimos hoy por los señores de esta comunidad, por los pescadores, que Dios les ayude en sus trabajos.

El día de la fiesta de la virgen inició con "Las mañanitas". Arreglaron la iglesia las mujeres encargadas, colocaron más flores, veladoras y la dejaron abierta desde la noche anterior. A las 3:00 de la mañana llegó un hombre adulto, ejidatario, pescador retirado, y fue a la iglesia. Me dijo que había dado gracias a la virgen por haberle permitido llegar con salud un año más en que celebraba su día. Diez minutos después llegaron dos mujeres de dos centros familiares diferentes, una llevaba un ciga$\mathrm{rro}^{11}$ y la otra dos cohetes. Me dijeron que eran "el despertador para la comunidad, ...así los que estén dormidos se despiertan y vienen a 'Las mañanitas' para la virgen", comentaron que no habían dormido, porque estaban preparando la comida para la fiesta desde la noche anterior en que había terminado el festejo ofrecido a las madres por el PRI.

A los pocos minutos de sonar el "despertador comunitario" comenzaron a llegar varios señores, algunos eran ejidatarios o pescadores de la UPPEEP. Llegaban en los vehículos de sus organizaciones. Otros lo hicieron en vehículos particulares, la mayor parte llegó caminando. Comenzaron a llegar señoras de andar presuroso, algunas de las catequistas y, en menor medida, varones adolescentes.

Quienes iban llegando a "Las mañanitas" se reunían por el parque; otros, hacia el atrio de la iglesia. Se encontraban con parientes y amigos, conversaban entre sí. Las mujeres conversaban acerca de cómo se despertaron, algunas decían que cuando oyeron los voladores ya estaban despiertas, otras que los voladores las despertaron. Después de esperar algunos minutos comenzaron a preguntar qué estaban esperando para entrar a la iglesia. Un hombre respondió que a la banda (de música), porque habían ido a buscarlos a El Pueblo. Al llegar los músicos, todos se congregaban a la entrada de la iglesia y juntos entraron cantando "Las mañanitas". Ocuparon las bancas. Algunos asistentes rezaron durante unos minutos, otros permanecieron sentados viendo hacia el altar. Luego fueron saliendo y cuando ya casi todos estaban afuera, algunas señoras se comunicaron con los conductores de los vehículos y, junto con los demás que 
aún estaban presentes, prepararon lo necesario para la misa, trasladaron la mesa de celebración, mantel, floreros, velas, sillas y la virgen al puerto de abrigo cercano.

Transformaron el puerto de abrigo en un nuevo escenario monumental, en un espacio ritual terrestre y marino que dio lugar a la celebración de la misa, teniendo como límite el mar, interceptado por los barcos y lanchas estacionados a la orilla. El aire fresco de la madrugada hizo sentir un poco de frío y algunas de las presentes llevaban puesta una toalla sobre la espalda ${ }^{12}$. Cuando aún estaba oscuro acomodaron todo: la mesa de celebración hacia el norte, a la orilla del mar, sillas orientadas de sur a norte, de tal manera que los feligreses veían hacia el norte. La virgen fue colocada en el barco elegido el día del primer gremio ${ }^{13}$, ataron la base de la imagen y le colocaron algunos floreros. Ella quedaba de frente a los feligreses, atrás del sacer- dote. El dueño del barco la observaba de cerca, bajo la penumbra de la madrugada. Su barco era el único que estaba adornado con flores y llevó, además de la virgen, al sacerdote, a las rezadoras y a los músicos. También estaba listo para salir a pescar, al terminar la fiesta de la virgen: ya tenía el hielo dentro de la embarcación. Los pescadores de los otros barcos inflaban globos y los colocaban en los suyos, para adornarlos. Al terminar la misa los asistentes abordaron las embarcaciones y pasearon con la virgen por el mar - acompañados por la banda de música y el sonido de los cohetes que tronaban - frente al asentamiento de su comunidad, luego retornaron y la llevaron en procesión hasta el local de la UPPEEP, lugar donde entró el gremio de ese día y realizaron el festejo.

La reunión ordinaria de los socios de la UPPEEP, posterior a la fiesta de la virgen, permitió constatar su capacidad organizativa y el funcionamiento

Figura 1

Intersecciones entre los ámbitos de la religión, política y actividades productivas para la fiesta de Fátima en El Puerto, Yucatán

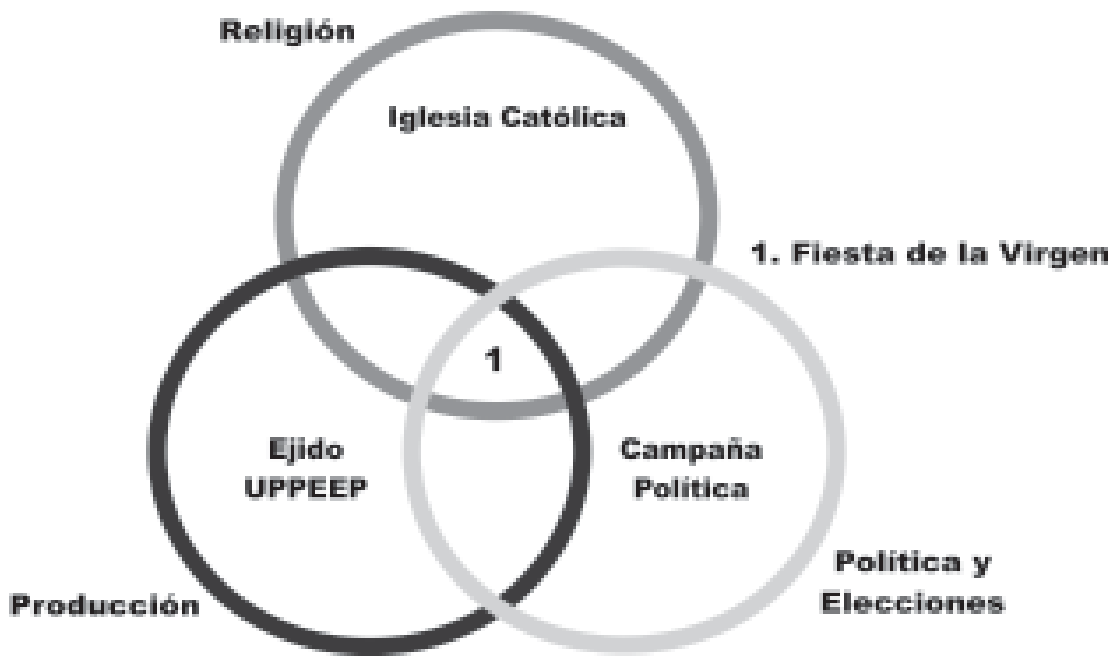


de la red de relaciones con la que cuentan. Para la organización de la fiesta realmente se dio la intersección de tres ámbitos: religión, producción y política, tal como podemos apreciar en la figura 1. Los miembros de la cooperativa que se encuentra en el ámbito productivo obtuvieron apoyo de los candidatos del PRI, del ámbito político, para celebrar la fiesta que es del ámbito religioso.

Los candidatos colaboraron ${ }^{14}$ y los socios se encargaron de transportar los apoyos hacia El Puerto. Los gastos restantes del festejo los cubrieron vía colaboración de cada encargado de barco. En la celebración hubo alegría y satisfacción. Uno de los socios dijo, en son de broma, que la fiesta de la virgen debería ser cada tres años, cuando fuera tiempo de "polilla", porque así los candidatos colaborarían y no saldría tan caro. Por su parte, los candidatos esperaban verse recompensados con los votos de los ciudadanos de la comunidad en la elección que efectuarían diez días después. Pocos en la comunidad sabían los esfuerzos involucrados para cristalizar la fiesta y en el ánimo comunitario general, la fiesta la organizó la UPPEEP.

En el entramado de la participación comunitaria existen lugares de relación y negociación restringidos. Un ejemplo es cómo establecen comunicación para la fiesta los encargados de la UPPEEP con las mujeres de los centros familiares, o con alguna de ellas, para organizar la comida para esta fiesta. El presidente de la cooperativa pesquera y la encargada de uno de los centros familiares eran esposos. Ella y otras mujeres tomaron su lugar para llevar adelante el gremio de los señores dado que ni él ni los otros asistieron el día que les tocaba. También una de las catequistas es hija de esta pareja. Ella participó activamente en la organización de la fiesta. Los tres se encontraban entre los veinte hombres y otras tantas mujeres más participativos de la comunidad. Este es uno de varios ejemplos de cómo las redes de relaciones comunitarias también están permeadas por lazos de parentesco, de vecindad y de amistad.

\section{Discusión y conclusiones}

Los resultados de la investigación aquí descritos ilustran la naturaleza y magnitud de la participación comunitaria. En ellos observamos que alrededor de la misma, hay un conocimiento más amplio sobre la vida comunitaria y su organización social, política y religiosa como lo señalan Villa Rojas (1946, 1987) y Brown (1993); caudal de costumbres de la vida cotidiana de la población manifestado en términos individuales, grupales, así como en un gran número de ámbitos o espacios de participación (Castillo, 2001).

Nuestros resultados también identifican que existen intersecciones entre los ámbitos de participación comunitaria y muestran que en la aparente simplicidad de una comunidad pequeña, opera un entramado de relaciones permeadas por el parentesco, la amistad y la vecindad, lo que repercute en la organización y toma de decisiones en torno de los eventos comunitarios.

Si bien Uphoff (1995) y Cernea (1995) sostienen que las agencias de desarrollo nacionales e internacionales han indicado las bondades de la participación comunitaria como eje transformador de la pobreza y la planeación del crecimiento. A nuestro perecer la concepción de participación comunitaria que subyace en dichos planteamientos es restringida, pues estos programas delimitan externamente lo que necesitan que la comunidad haga, sin valorar el acervo cultural de las comunidades, una serie de eventos y formas de participar complejas y ricas pero 
que, al no ser lo suficientemente visibles, no son debidamente valoradas. La falta de atención a estos señalamientos, entre otros, ha contribuido a que las agencias ligadas con el desarrollo no hayan cumplido satisfactoriamente sus objetivos y metas.

A pesar del medio siglo transcurrido, sigue vigente la recomendación del Community Services Staff (1953), sobre la importancia de realizar estudios de base para conocer la situación económica y cultural de los grupos de interés. En el mismo sentido, Chanan (1993) identifica que existen una serie de impedimentos para la participación comunitaria. Consideramos que es necesario retomar los planteamientos de los autores mencionados, pero que deberán ser reorientados en términos de los deseos y necesidades de las comunidades participantes en relación con el proyecto, la planeación, toma de decisiones y manejo de información. Por otra parte, es importante el llamado de Smith (1997) en términos de reconocer e incentivar la colaboración de todos los interesados mediante la promoción del cambio de actitudes.

Conocer los entornos que la comunidad construye para la participación comunitaria, los arreglos y las transformaciones que en ellos se realizan permite explicar el entramado de esa participación y la cultura que subyace a nivel comunitario. Un entorno construido para una actividad no necesariamente implica que sea exclusivo, sino que da lugar a transformaciones o reconstrucción del entorno según las necesidades de los participantes. Esta versatilidad les permite realizar las celebraciones comunitarias de acuerdo con los calendarios y usar los recursos de diversa índole que los participantes tienen, sea de manera personal o a través de las relaciones que establecen desde sus organizaciones. Este conjunto de elementos propicia las condiciones necesarias para la cohesión social.

En la participación comunitaria convergen diversos ámbitos. Uno de ellos, que usualmente aglutina a la mayor parte de la comunidad, es el religioso. En este estudio damos cuenta sobre cómo gran parte de las actividades comunitarias, independientemente de los lugares donde fueran realizadas, -iglesia, calles, hogares, comisaría, puerto de abrigo, cancha de baloncesto- estaban vinculadas con la religión católica. Si bien pareciera que en el ámbito religioso el orden ya está establecido, la comunidad tiene margen de maniobra y la usa de acuerdo con sus necesidades y momentos.

\section{Notas}

* Laboratorio de Investigación y Participación Comunitarias, Departamento de Ecología Humana, Centro de Investigación y de Estudios Avanzados del IPN, Unidad Mérida, http://www.mda.cinvestav.mx

${ }^{1}$ Expresión local de la gente de El Puerto para referirse a la época de elecciones, a la que suelen nombrar también como política, "polilla".

${ }^{2}$ En un recuadro de la tercera parte de este informe, que trata sobre las formas de revitalizar la capacidad institucional, describen un caso en Perú e indican que la participación popular permite mejorar el régimen de los derechos de propiedad y la solución de controversias en ese país.

${ }^{3}$ Trabajo financiado por la Fundación europea para la mejora de las condiciones de vida y de trabajo. Fue preparado para una conferencia europea en Dublín en septiembre de 1992 sobre "Acción ciudadana: participación de las personas a nivel local". ${ }^{4}$ Este autor tiene como formación de origen la arquitectura y ha buscado a lo largo de sus estudios comprender cómo se da la interacción entre hombre y ambiente. Su trabajo nos permite constatar la necesidad de explorar de manera multidisciplinaria e interdisciplinaria las áreas de intersección de un problema, como una forma de búsqueda de respuesta a las interrogantes. 
${ }^{5}$ Este estudio se realizó entre 1990 y 1991 por un equipo de colaboradores, del cual formé parte.

${ }^{6}$ Llamaremos de esta manera al lugar donde efectuamos la investigación. Se trata de una manifestación de respeto a la vida cotidiana de la comunidad, guardando el anonimato y la privacidad de sus moradores. De igual manera, al referirnos a informantes que proporcionaron datos, se indicará un breve perfil de la persona, pero no sus nombres.

${ }^{7}$ Llamamos ciclo comunitario anual al periodo de doce meses, un año natural, durante el cual tienen lugar diversos eventos de participación comunitaria. Consideramos que esta es la unidad básica para comprender la manera, lugares, escenarios, personas y relaciones presentes en la vida comunitaria.

${ }^{8}$ Observamos 269 eventos durante el ciclo comunitario anual.

${ }^{9}$ Chapear es la forma local para referir que limpian las yerbas que crecen en algún lugar.

${ }^{10}$ Empacadora y Congeladora Pescormex, S.A. de c.V.

${ }^{11}$ Esta mujer es responsable de uno de los centros familiares, es la cuarta más participativa, realiza una intensa labor en actividades relacionadas con la comunidad. Me explica que se fastidiaron de tener que estar rogándole a los señores para que truenen los voladores. No fuma y me dice que ella decidió aprender a tronar los voladores, así no tienen que depender de si hay señores para que los truenen, si no hay ella lo hace; es la única mujer que ha aprendido a hacerlo.

${ }^{12}$ Esta es una práctica local común y recuerda el uso del rebozo, aunque las toallas son más cortas.

${ }^{13}$ Ese día, solamente hubo un hombre adulto participante en la misa, situación que pareció extraña, dado que solamente había mujeres y niños. Al finalizar la misa el enigma se despejó. El sacerdote anunció que harían la rifa del barco para la virgen. Para ello estaba ese hombre adulto en la iglesia, era un pescador que representaba a su organización, para anunciar a sus compañeros "a quién había elegido la virgen para ir con él en su barco". El nombre del pescador que resultó electo motivó expresiones de asombro, debido a que el año pasado también había resultado electo y no había cumplido. Ante esta situación el sacerdote exclamó: “¡Ni modo, la virgen ya dijo que quiere ir con él!"

${ }^{14}$ Los candidatos dieron $15 \mathrm{~kg}$ de pollo, mil jugos y la música para amenizar la fiesta en el local de la cooperativa.

\section{Bibliografía}

Aboites, L., 1995, Norte precario: poblamiento y colonización en México (1760-1940), El Colegio de México, México.

Banco Mundial, 1997, Informe sobre el Desarrollo Mundial. El estado en un mundo en transformación. Oxford University Press, Wahington D.C., EUA.

—, 2000/2001, World Development Report 2000/200. Attacking

Poverty. Oxford University Press, Wahington D.C., EUA.

Boas, F., 1911, Handbook of American Indian Languages, en Taylor, S. J. y R. Bodgan, 1996, Introducción a los Métodos Cualitativos de Investigación, Paidós Básica, Barcelona.

Brown, D. F., 1993, Yucatec Maya Settling, Settlement and Spatiality, Ph.D. Dissertation in Anthropology, University of California, Riverside, EUA.

Burgman, H. y J. Ooijens, 1989, La participación de la mujer en el desarrollo rural, Juan Pablos, México.

Castillo, M. y F. Dickinson, 1994, "Estado, ecología y estrategias de sobrevivencia en un municipio rural de Yucatán" en E. Ortiz (coord.) Economía, Teoría y Práctica (2):123-132.

Castillo M. T., 2001, Relaciones de género en los ámbitos de participación comunitaria de un pueblo de la costa yucateca, Tesis de Doctorado en Antropología Social, Universidad Iberoamericana, México.

Centro de Salud, 1997, Microdiagnóstico de El Puerto, El Puerto.

Cernea, M., (coord.) 1995, Primero la gente. Variables sociológicas en el desarrollo rural, Fondo de Cultura Económica/Economía Contemporánea, México.

Chanan, G., 1993, Acción comunitaria Localy Política Social. Documento para Debate, Fundación Europea para la mejora de las condiciones de vida y de trabajo, Luxemburgo.

Community Services Staff, 1953, "Method of Obtaining Community Participation in Self-Help Activities", Technical Cooperation Administration. Department of State, Mimeo, EUA. Creswell, J. W., 1998, Qualitative inquiry and research design. Choosing among five traditions, Sage, Thousand Oaks, Ca., EUA. Denzin, N. K. y Lincoln, Y. S., 1994, "Introduction. Entering the Field of Qualitative Research" in: Denzin, N. K. y Lincoln, Y. S. (eds.) Handbook of qualitative research, Sage, Thousand Oaks, Ca., EUA.

Dickinson, F. y J. Ortega, 1994, Ecological, Sociological and Health Assessment (Mexico). Phase II. Final Technical Report. Centro de Investigaciones y de Estudios Avanzados del IPN-Unidad 
Mérida/Centro de Investigaciones Regionales "Dr. Hideyo Noguchi"- UADY, Mérida, Yucatán.

Fals Borda, O., 1988, Knowledge and People Power, Indian Social Institute, Nueva Delhi.

Funaro, R., 1997, "La marcha del desarrollo". Desarrollo de Base, EUA.

Gamio, M., 1922, La población del valle de Teotibuacán, 2 Tomos, México.

Hall, E. T., 1972, La dimensión oculta, Siglo xxi, México.

Hollahan Ch., 2000, Psicología Ambiental. Un enfoque general, Editorial Limusa, Noriega Editores, 467 pp.

Hawley, W. D. and F. Wirt (eds.) 1974 The Search for Community Power, Prentice Hall, Inc., Englewood Cliffs, Nueva Jersey, EUA. Hoffman, O. y F. Salmerón, 1997, "Introducción. Entre representación y apropiación, las formas de ver y hablar del espacio" en O. Hoffman y F. Salmerón (coords.) Nueve estudios sobre el espacio. Representación y formas de apropiación, CIESASORSTOM, México.

Lawrence, D. and S. Low, 1990, "The Built Environment and Spatial Form” Ann. Rev. Anthropol. (19):453-505.

Malinowski, B., 1973, Los Argonautas del Pacífico Occidental, Península, Barcelona, España, edición original inglesa 1922. Nahmad, S., 1995, “Introducción” en M. Cernea (coord.) Primero la gente. Variables sociológicas en el desarrollo rural, Fondo de Cultura Económica/Economía Contemporánea, México. Organización Panamericana de la Salud y Organización Mundial de la Salud, 1978, Extensión de la cobertura de servicios de salud con las estrategias de atención primaria y participación de la comunidad. Resumen de la situación en la Región de las Américas, Documento Oficial Núm. 156, Washington, D.C., EUA. Organización Panamericana de la Salud y Organización Mundial de la Salud, 1984 Participación de la comunidad en la saludy el desarrollo en las américas. Análisis de estudios de casos seleccionados, Publicación Científica Núm. 473, Washington, D.C., EUA.

Ortega, J. y F. Dickinson, 1991, Monografía de Sinanché. Informe de Investigación, UADY-CINVESTAV, Mérida, Yuc., México.

Pearse, A. y M. Stiefel, 1980, "Participación popular: un enfoque de investigación” en Socialismo y Participación, núm. 3. Citado en: Pliego, F. 1995, Participación comunitaria y cambio social, Tesis de Doctor en Ciencias Sociales con especialidad en Sociología, El Colegio de México, México.

Pliego, F., 1995, Participación comunitaria y cambio social, Tesis de Doctor en Ciencias Sociales con especialidad en
Sociología, El Colegio de México, México.

Polsby, N. W., 1974, "El estudio del poder en la comunidad" en Enciclopedia Internacional de las Ciencias Sociales, Aguilar. Tomo 2, España.

Rahnema, M., 1997, "Participation” in W. Sachs (ed.) The Development Dictionary. A Guide to Knowledge as Power, wup, Johannesburgo.

Rapoport, A., 1990, "Systems of Activities and Systems of Settings" in S. Kent (ed.) Domestic Architecture and the Use of Space: an Interdisciplinary Cross-cultural Study, Cambridge University Press, Cambridge, EUA.

—, 1990a, The Meaning of the Built Environment. A nonverbal Communication Approach, The University of Arizona Press, Tucson, EUA.

—, 1994, "Prólogo" en M. Amerlinck y F. Bontempo El entorno construido y la antropología: introducción a su estudio interdisciplinar, Centro de Investigaciones y Estudios Superiores en Antropología Social, México.

Redfield, R. y A. Villa Rojas, 1934, Chan Kom. A Maya Village, The University of Chicago Press, Chicago y Londres.

Rifkin, S., F. Mullery W. Bichmann, 1988, "Primary Health Care: On Measuring Participation” Soc. Sci. Med. 26(9):931-940.

SAHOP, s.f., Formación de promotores en desarrollo y participación de la comunidad. Guía para el instructor, Secretaría de Asentamientos Humanos y Obras Públicas, México.

Schnell, I., 1999, "Segregating Practices in Social Space" in Aguilar, A. e I. Escamilla (eds.) Problems of Megacities: Social Inequalities, Environmental Risk and Urban Governance, Universidad Nacional Autónoma de México, México.

Smith, K., 1997, "Encuentros: Donde el Norte encuentra al Sur”. Desarrollo de Base, EUA.

Stacey, M., 1969, The myth of community studies" The British Journal of Sociology Vo. 20(3):134-147.

Taylor, S. J. y R. Bodgan, 1996, Introducción a los Métodos Cualitativos de Investigación, Paidós Básica, Barcelona.

Uphoff, N., 1995, “Adaptar los proyectos a la gente" en M. Cernea (coord.) Primero la gente. V ariables sociológicas en el desarrollo rural, Fondo de Cultura Económica, México.

Villa Rojas, A., 1946, "Los mayas del actual Territorio de Quintana Roo" en Enciclopedia Yucatanence, Tomo vi:31-62, Edición oficial del Gobierno de Yucatán, Yucatán, México. —, 1987, Los Elegidos de Dios. Etnografía de los mayas de Quintana Roo, Instituto Nacional Indigenista, México. 